\title{
MÉTODOS Y TÉCNICAS DE INVESTIGACIÓN PARA LAS CIENCIAS SOCIALES
}

\author{
Miguel Ángel Medina Martínez* \\ mmedinamartinez@yahoo.com.mx \\ Guillermo Briones \\ Ed. Trillas
}

El autor desarrolla con suficiente exhaustividad, pero sin exagerar en la amplitud, los temas de mayor importancia en torno a los aspectos cuantitativos y cualitativos de la investigación en las ciencias sociales para solventar los muy diversos problemas y a veces situaciones dilemáticas que se presentan a lo largo del proceso investigativo. Se trata de un libro en el que pese a la extensión numérica de los capítulos se abordan sucintamente todos y cada uno de los temas principales en torno a la investigación en las ciencias sociales.

Los grandes rubros que contiene el libro están organizados en una perspectiva didáctica que va de lo general a lo específico y que facilita -por ende- su lectura y comprensión:

1.El Proyecto de Investigación, que incluye los conceptos más generales y básicos en torno a la preparación del proyecto, la clarificación y determinación de las variables e hipótesis, algunos tipos de medición, así como la importancia de su validez y el grado de confiabilidad.

2.En las Investigaciones Cuantitativas propiamente dichas, las características que la definen, la diferencia entre investigaciones y diseños experimentales y cuasiexperimentales.

3.La Encuesta Social, los diferentes tipos de encuestas, los diseños de las mismas, así como todo lo relativo a la elaboración del cuestionario: su estructura, las pruebas previas, la codificación y el informe final.

4.A partir de la sección titulada Probabilidad y Teoría de la Muestra, comienza el autor a referirse específicamente a los aspectos más específicos de la estadística y probabilidad: los tipos de muestreo, de distribución, de errores y diseños muestrales.

5.En Análisis Descriptivo abunda acerca de las nociones y la diferenciación entre razones, proporciones, porcentajes y tasas, las distribuciones de frecuencias, y las medidas de tendencia central, de dispersión y de concentración.

6.Las Medidas de Asociación y de Correlación son tratadas desde la comparación de porcentajes entre variables nominales y ordinales, el coeficiente ETA, la correlación de Pearson y de Spearman. 
7.La sección del Análisis Inferencial, es abordado en dos secciones: las pruebas paramétricas (para aprender a determinar la región de rechazo de Ho., las relaciones entre el tamaño de la muestra, el nivel de significación y el poder de la prueba) y las no paramétricas (muestras relacionadas o pareadas, muestras independientes y análisis de varianza de Kruskall-Wallis).

8.Análisis Multivariable I contiene lo relacionado con la regresión lineal simple, múltiple, las pruebas de significación estadística, así como el análisis de la varianza (ANOVA y MANOVA) y covarianza.

9.Análisis Multivariable II aborda otras técnicas de análisis multivariable tales como la correlación canónica la regresión logística, los modelos loglineales, los análisis d conglomerados, de senderos, entre otros.

10. Métodos Prospectivos de Investigación es la última parte y está consagrada a desentrañar la naturaleza y los objetivos de la misma, al método Delphi, al análisis estructural, al método del juego de actores y al método de impacto cruzado y la construcción de escenarios deseables.

Llama la atención que, desde el principio, el autor haga un distingo entre dos tipos de investigación que obedecen a diferentes supuestos epistemológicos y que se manifiestan en distintos campos y niveles de aplicación, a saber: la investigación cuyo objeto de análisis lo constituyen las totalidades globales en el marco de las estructuras y procesos históricamente determinados y concretos.

El otro tipo de investigación es aquélla que tiene por objeto el análisis de variables que, en su tratamiento estadístico, quedan separadas de la totalidad compleja y concreta.

Por último y para reforzar aún más la idea de la estructura didáctica en la que fue concebido y publicado este libro, es importante señalar que cada explicación de los conceptos, métodos, diseños, análisis o estrategias utilizados en el libro, está acompañada de sus respectivos ejercicios, en los cuales se pueden poner en práctica los conocimientos adquiridos. 